Research Paper

\title{
Numerical Modeling of Nano-powder Synthesis in a Radio-Frequency Inductively Coupled Plasma Torch
}

\author{
Min Young Hur ${ }^{a}$, Donggeun Lee ${ }^{\mathrm{b}}$, Sangsun Yang ${ }^{\mathrm{c}}$, and Hae June Lee ${ }^{\mathrm{a} *}$ \\ ${ }^{a}$ Department of Electrical and Computer Engineering, Pusan National University, Busan 46241, South Korea \\ ${ }^{b}$ School of Mechanical Engineering, Pusan National University, Busan 46241, South Korea \\ ${ }^{c}$ Powder and Ceramics Division, Korea Institute of Materials Science, Changwon 51508, South Korea
}

Received January 12, 2018; accepted January 26, 2018

\begin{abstract}
In order to understand the mechanism of the synthesis of particles using a plasma torch, it is necessary to understand the reaction mechanisms using a computer simulation. In this study, we have developed a simulation method to combine the Lagrangian scheme to follow microparticles and a nodal method to treat nanoparticles categorized with different particle sizes. The Lagrangian scheme includes the Coulomb force which affects the dynamics of larger particles. In contrast, the nodal method is adequate for the nanoparticles because the charge effect is negligible for nanoparticles but the number of nanoparticles is much larger than that of microparticles. This method is helpful to understand the dynamics and growth mechanism of micro- and nano-powder mixture observed in the experiment.
\end{abstract}

Keywords: RF-ICP thermal plasma, Micro- and nano-powder synthesis, Lagrangian scheme, Nodal method

\section{Introduction}

The inductively coupled plasma (ICP) torch is widely used for nano-powder synthesis, being characterized by high enthalpy, high reactivity, high process purity, large plasma volume, and long resident time due to the comparatively low plasma velocity [1-3]. The experimental measurement in an ICP torch has a limitation because of the high temperature [4]. In order to understand the mechanism of the generation of particles for an intended size, it is necessary to understand the physics inside of the ICP torch using a computer simulation. Especially, the simulation of particle dynamics and transport in the ICP torch is not widely introduced in the plasma society. The plasma in the ICP torch system is a kind of thermal plasma, which is calculated by computational fluid dynamic models [5-8]. The simulation of the transport and the growth of the powder in the plasma is also conducted in some studies. The one-dimensional nodal method is used for fabrication of silicon-based functional nanoparticles to clarify the growth mechanism [9]. The nodal method is based on the concept of nodal discretization [10]. The moment method which is solving the aerosol general dynamic equation (GDE) is used for platinum nanoparticle formation [11]. The two-dimensional nodal method for the study of the synthesis of Si nano-powders [4]. These

*Corresponding author

E-mail: haejune@pusan.ac.kr methods mentioned above do not calculate the Coulomb force among powders which are charged negatively in the plasma [12]. The Lagrangian simulation is used for electrospray droplets dynamics to calculate the Coulomb force $[13,14]$. However, this particle simulation in the Lagrangian scheme needs long calculation time compared with the nodal method if the number of particles is large.

In this study, we present the method to combine the plasma chamber simulation and the particle dynamics calculation. A two-dimensional fluid simulation is used for the former, and the combined method of a nodal analysis and the Lagrangian scheme is newly added for the latter. In this system, the precursor powder is not fully evaporated but only partially, and the number of the generated nanoparticles by nucleation is also very large. In order to take advantage of each method, we have developed a method to combine the Lagrangian scheme and the nodal method concerning different particle sizes. The Lagrangian scheme which follows every microparticle has the advantage to consider the Coulomb force influencing their motion within a Debye length. On the other hand, the nanoparticles are calculated by nodal method because they are almost neutral and the number of them is much larger than microparticles. The charging mechanism, simulation methods, and simulation conditions are explained in Sec. 2 followed by the simulation results for the variation of quenching gas in Sec. 3. Finally, the conclusion is presented in Sec. 4. 


\section{Numerical Modeling}

\section{Simulation Method}

Figure 1 shows the simulation chart which is combining the plasma chamber simulation and particle dynamics simulation. The RF-ICP torch is calculated by DCPTUN (DC Plasma Torch in Unstructured Grid System) code developed by Park et al. [15]. It is based on these assumptions that the plasma is optically thin, electrically neutral, turbulent and a local thermodynamic equilibrium (LTE). It describes the plasma flow in the framework of magnetohydrodynamic (MHD) equations using semiimplicit method for pressure linked equation revised (SIMPLER) algorithm [16] in conjunction with the $K-\varepsilon$ turbulence model [17].

The growth and dynamics of microparticles are calculated by the Lagrangian scheme which follows every particle using Newton's equation of motion and considering the variation of the size and the charge of each particle. The calculation of microparticle dynamics includes the Lorentz force, drag force, gravity, stochastic force and the Coulomb force. The growth of microparticles is considering the condensation, evaporation. The transport of monomer is calculated by a convection-diffusion equation to consider the gas phase evaporation. And the growth and dynamics of nanoparticles are calculated by the two-dimensional nodal method which calculates at discrete nodes [9]. The dynamics of nanoparticles include the drag force, gravity, and stochastic force. The growth of nanoparticles is considering the condensation, evaporation, nucleation, and coagulation.

The significant difference of the nodal method from the Lagrangian scheme is the Coulomb force. For nanoparticles, the Coulomb force is neglected because the charge of nanoparticles is very small with the reason as follows.

The charge of nanoparticles can be calculated by following equation [18].

$$
\frac{\mathrm{d} q}{\mathrm{~d} t}=I_{e} \exp \left(\frac{q}{C_{d} T_{e}}\right)+I_{i}\left(1-\frac{q}{C_{d} T_{l}}\right)
$$

$q, I, C_{d}, T$ is the charge of nanoparticle, current from plasma to the nanoparticle, capacitance of nanoparticle, and temperature, respectively. The subscript $e, i$ mean the electron and ion. The current is depend on the plasma density which estimated by the Saha equation [19]. At this time, the gas is composed of only singly ionized ions, electrons, and neutral particles. The same internal function as that of Drellishak's paper [20] is used for the calculation of the Saha equation. The full charging time is calculated roughly as follow [18].

$$
\tau \approx \frac{C_{d} T_{i}}{I_{e 0}}
$$

The charging time is much shorter than the residence time of the precursor particles at $10,000 \mathrm{~K}$, and much longer at $500 \sim 1,500 \mathrm{~K}$. The charging time becomes similar to the residence time at $3,000 \mathrm{~K}$.

In the ICP torch, nanoparticles are mainly generated by nucleation where the plasma temperature is low which results in very long charging time compared with the residence time of the nanoparticles. Therefore, the charging effect of the nanoparticle is negligible, which validate the nodal method in low-temperature regime.

\section{Simulation conditions}

Figure 2 shows the simulation domain. The chamber pressure is $1 \mathrm{~atm}$. The flow rates are $15 \mathrm{slpm}(\mathrm{Ar}), 85 / 10$ slpm $\left(\mathrm{Ar} / \mathrm{H}_{2}\right)$ and 5 slpm (Ar) for the central gas, sheath gas, and carrier gas, respectively. The flow rate of quenching gas (Ar) varies from 50 to $250 \mathrm{slpm}$. The RFICP power is $20 \mathrm{~kW}$ with a driving frequency of $3 \mathrm{MHz}$. The feeding nozzle position is $11.5 \mathrm{~cm}$. The time step of nanoparticle calculation is $10 \mu \mathrm{s}$. The mass flow rate of
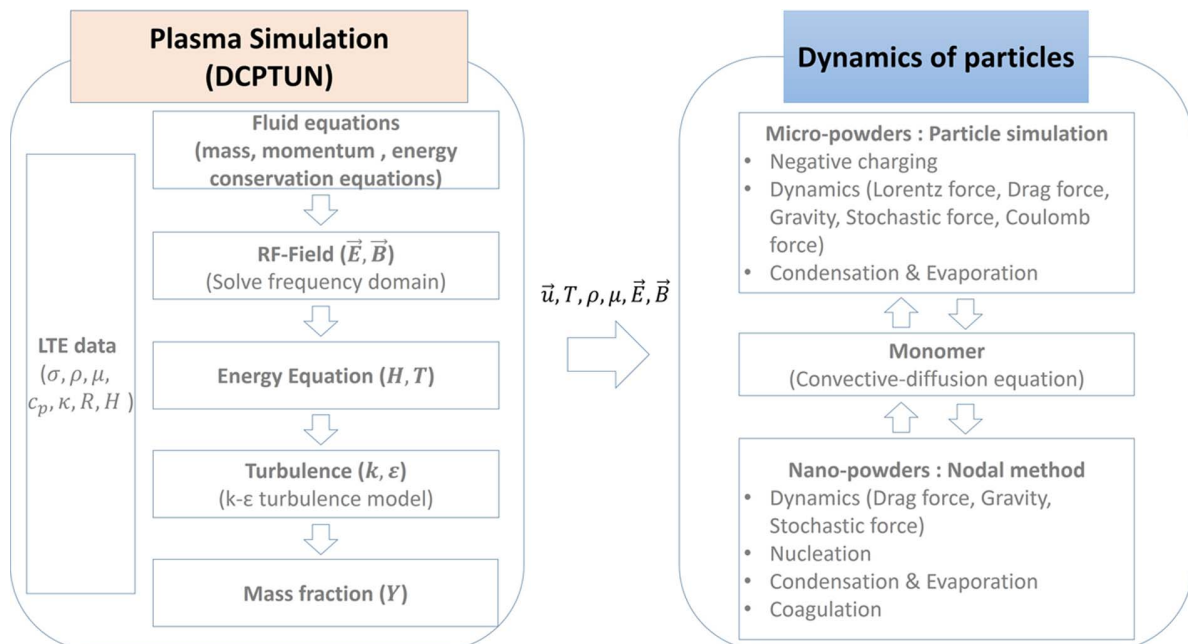

Figure 1. Simulation scheme used in this study is composed of a two-dimensional plasma fluid simulation and two methods to calculate the dynamics of microparticles and nanoparticles together. 


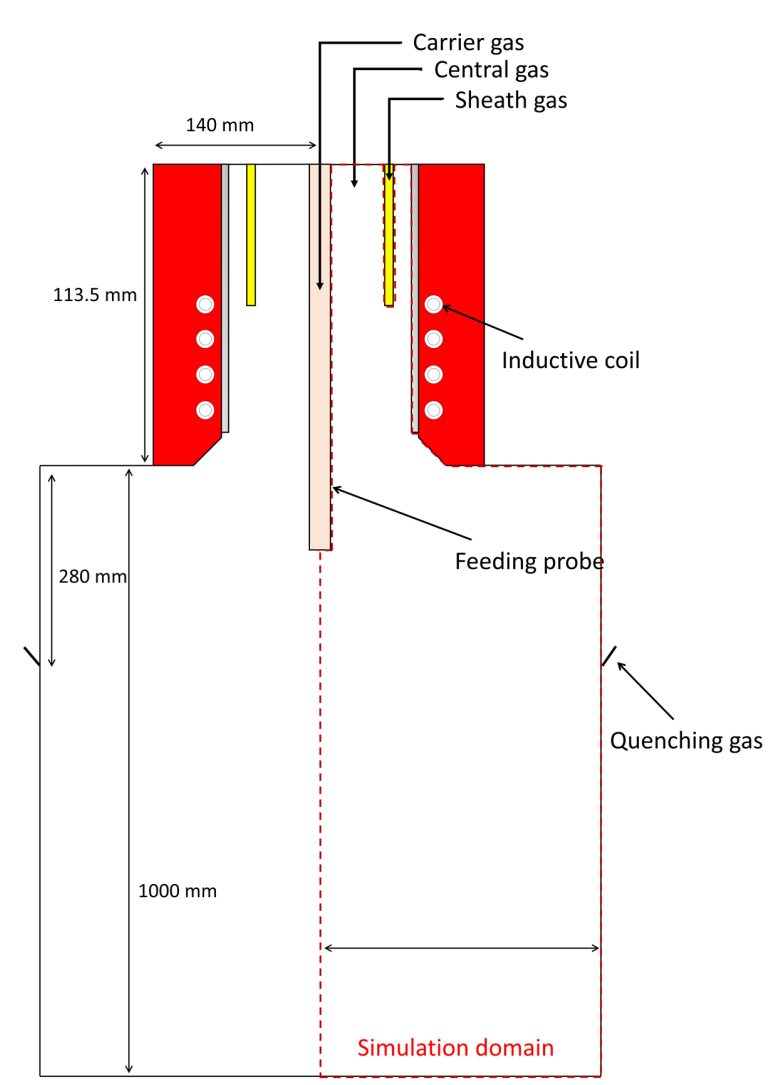

Figure 2. The RF-ICP torch system considered in this study.

silver precursor is $0.001 \mathrm{~kg} / \mathrm{m}^{3}$. The initial size of nanoparticles has Gaussian distribution with an average diameter and a deviation of $5.0 \mu \mathrm{m}$ and $0.2 \mu \mathrm{m}$. The volume of the first node is 10 times larger than the monomer volume, and the volume of the next node is multiplied by a factor of 1.8 to the volume of the previous node. The number of nodes is set to be 33 , which diameter ranges from 0.71 to $400 \mathrm{~nm}$.

\section{Results}

Figure 3 shows the profiles of the plasma temperature and the axial plasma velocity from the feeding probe to the downstream area. The flow rates of the quenching gas are $50 \mathrm{slpm}$ (top figures) and $250 \mathrm{slpm}$ (bottom). The precursor particles passing through the feeding probe are evaporated by the high temperature plasma, up to $2,400 \mathrm{~K}$. The boiling temperature of silver is $2,435 \mathrm{~K}$ which is slightly higher than the maximum plasma temperature. It means that the precursor particles are evaporated slowly in a state of liquid rather than a liquid-gas state as shown in Fig. 4.

Figure 4(a) shows the size distribution of the particles along the axial position. The precursor powders move following the plasma flow and pass through the hightemperature region. The precursor powders with a diameter of $5 \mu \mathrm{m}$ evaporate at the high-temperature region at $\mathrm{z}=350 \mathrm{~mm}$ and decrease its size to $3.8 \mu \mathrm{m}$ in the region from $\mathrm{z}=300$ to $\mathrm{z}=400 \mathrm{~mm}$. This evaporation becomes the
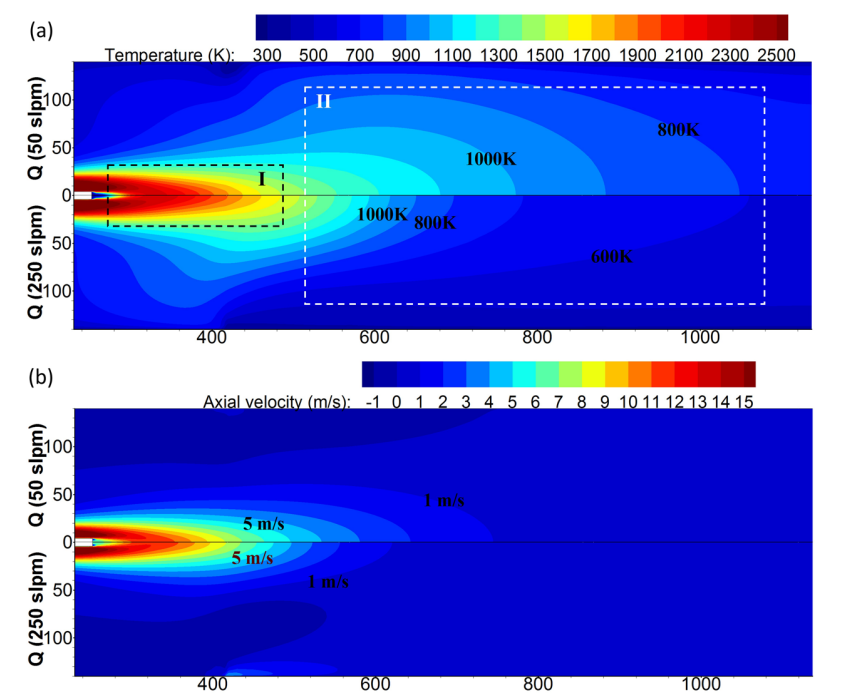

Figure 3. The profiles of the plasma temperature and the axial plasma velocity from the feeding probe to the downstream area. The flow rates of the quenching gas are $50 \mathrm{slpm}$ (top) and 250 slpm (bottom).
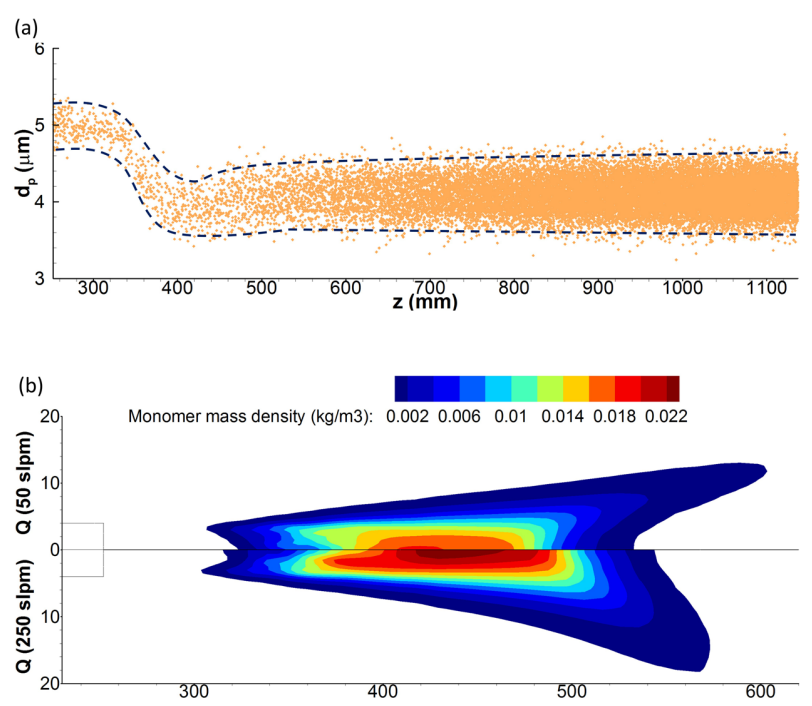

Figure 4. Shown are (a) the size distribution of the particles along the axial position and (b) the profiles of the monomer mass density with a flow rate of the quenching gas of $50 \mathrm{slpm}$ (top figures) and $250 \mathrm{slpm}$ (bottom).

source of the monomer as shown in Fig. 4(b). The monomers are generated by the evaporation of the precursor particles from $\mathrm{z}=300$ to $\mathrm{z}=400 \mathrm{~mm}$ and diffuse along the plasma flow. The maximum monomer mass density exists at $\mathrm{z}=430 \mathrm{~mm}$. The reason for the decreasing mass density of monomer after $\mathrm{z}=430 \mathrm{~mm}$ despite the slowed-down plasma flow is the consumption of monomer by the condensation and the nucleation.

Figure 5(a) shows the size distribution of precursor particles with the variation of the flow rate of the quenching gas. The average diameter of the precursor particles passing through the hot temperature region decreases because of the evaporation process. The higher flow rate of the quenching gas results in the smaller average diameter because the plasma temperature is high as the flow 

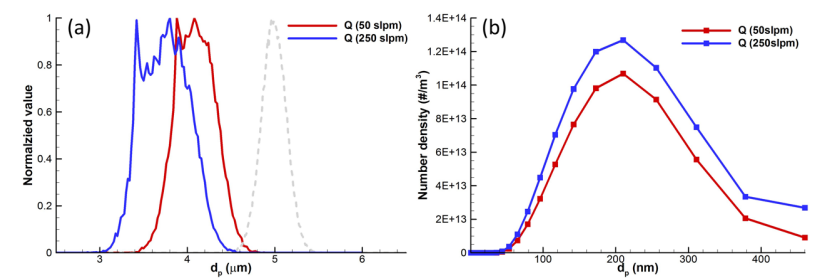

Figure 5. The size distributions of (a) microparticles and (b) nanoparticles are observed with the variation of the flow rate of the quenching gas. The dotted gray line is the size distribution of the initially feeded precursor powders.

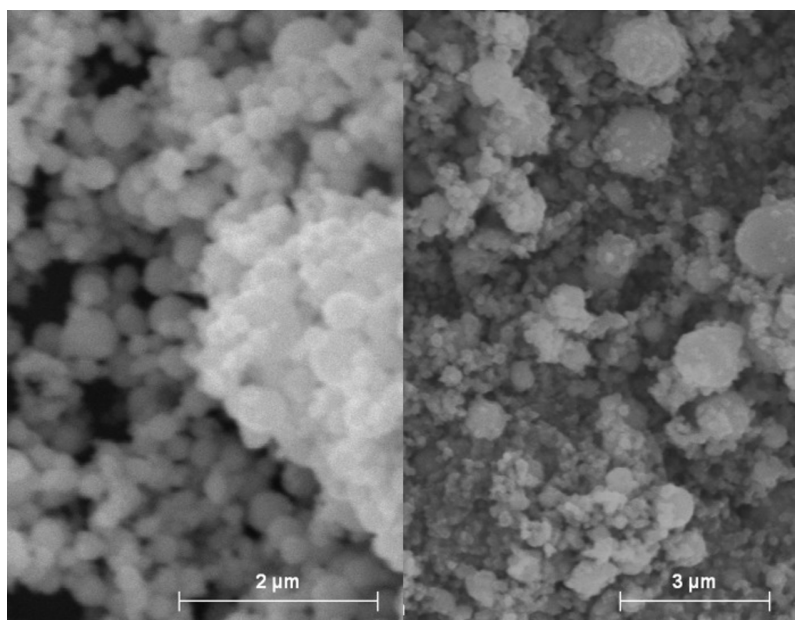

Figure 6. SEM images of stainless steel powders after RF-ICP torch treatment.

rate of quenching gas increases at the $\mathrm{z}$ range 300 to $410 \mathrm{~mm}$. Figure 5(b) shows the size distribution of nucleated nanoparticles with the variation of the flow rate of the quenching gas. The average diameter of the nucleated nanoparticles is about $200 \mathrm{~nm}$, and the nanoparticles grow up to $400 \mathrm{~nm}$ by coagulation process. The total amount of nanoparticles is larger with a higher flow rate of the quenching gas. A large amount of quenching gas decreases plasma temperature in region I shown in Fig. 3(a), but the plasma temperature in region II increases slightly with the flow rate of $250 \mathrm{slpm}$ compared with $50 \mathrm{slpm}$ because of turbulence effect near region II.

Figure 6 shows experimental observation of the considered system in our. As shown in the figure, there are large size particles with a diameter of $4 \sim 5 \mu \mathrm{m}$ and small size nanoparticles of which diameter is about $200 \sim 300 \mathrm{~nm}$. The tendency of the simulation results agrees well with the experimental observation.

\section{Conclusions}

We simulated the micro- and nano-powder synthesis in RF-ICP thermal plasma system using a two-dimensional fluid simulation code, DCPTUN, which analyzes the thermal plasma. For particles, a combination of a particle method (for microparticles) and a nodal method (for nanoparticles) are utilized to explain the behavior and growth of nanoparticles in the ICP torch. The precursor microparticles are injected from the feeding probe and evaporate partially. The simulation of microparticles in the Lagrangian scheme calculates the Coulomb force among negatively charged precursor particles. However, the Coulomb force is not effective in the considered cases because the dominant force for the precursor powder is drag force. In contrast, the nucleation nanoparticles cannot obtain the charge because they are generated at low temperature. It is easy to calculate nucleation nanoparticles using the nodal method because the number of nucleation nanoparticles is large. The combined method clarifies the generation of micro- and nano-powder mixture. The precursor powder evaporates partially to generate the smaller micro-powder. Nucleation process generates the nanoparticles which grow up to $400 \mathrm{~nm}$ by coagulation and condensation. The increase of the quenching gas increases the weight ratio of nano-power because the plasma temperature is high where the precursor powder evaporates.

The effect of Coulomb force does not stand out in this system. Some experiments observed the positively charged nanoparticles in the plasma [22-24]. However, the mechanism of positively charging is unknown. If the nanoparticles obtain the positive charge, they can grow by coagulation with negatively charged nanoparticles. Future work will clarify the effect of positively charged nanoparticles and Coulomb force.

\section{Acknowledgments}

This work was supported by the National Research Council of Science \& Technology (NST) grant by the Korea government (MSIP) (No. CAP-12-6-KIMS) and by BK21PLUS Creative Human Resource Development Program for IT Convergence.

\section{References}

[1] J. H. Seo and B. G. Hong, Nucl. Eng. Technol. 44, 9 (2012).

[2] M. I. Boulos, Pure Appl. Chem. 68, 1007-1010 (1996).

[3] M. K. Mun, W. O. Lee, J. W. Park, D. S. Kim, G. Y. Yeom, and D. W. Kim, Appl. Sci. Converg. Technol. 26, 164-173 (2017)

[4] V. Colombo, E. Ghedini, M. Gherardi, P. Sanibondi, and M. Shigeta, Plasma Sources Sci, Technol. 21, 025001 (2012).

[5] A. B. Murphy, J. Phys. D: Appl. Phys. 34, R151-R173 (2001).

[6] A. Gleizes, J. J. Gonzalez, and P. Freton, J. Phys. D: Appl. Phys. 38, R153-R183 (2005).

[7] J. P. Trelles, J. V. R. Heberlein, and E. Pfender, J. Phys. D: Appl. Phys. 40, 5937-5952 (2007).

[8] M. Hur and S. H. Hong, J. Phys. D: Appl. Phys. 35, 1946-1954 (2002).

[9] M. Shigeta and T. Watanabe, J. Phys. D: Appl. Phys. 40, 24072419 (2007).

[10] A. Prakash, A. P. Bapat, and M. R. Zachariah, Aerosol Sci. Technol. 37, 892-898 (2003).

[11] M. Shigeta and T. Watanabe, J. Appl. Phys. 103, 074903 (2008).

[12] A. Douglass, and V. Land, L. Matthews, T. Hyde, Phys. Plasma 18, 083706 (2011).

[13] J. Grifoll and J. Rosell-Llompart, J. Aerosol Sci. 47, 78-93 (2012). 
[14] A. K. Arumugham-Achari, J. Grifoll, J. Rosell-Llompart, and J. Aerosol Sci. 65, 121-133 (2013).

[15] J. M. Park, K. S. Kim, T. H. Hwang, S. H. Hong, and IEEE T. Plasma Sci. 32, 479-487 (2004)

[16] S. V. Patankar, Numerical heat transfer and fluid flow, Hemisphere (1980).

[17] B. E. Launder and D. B. Spalding, Comput. Methods Appl. Mech. Eng. 3, 269-289 (1974).

[18] M. A. Lieberman and A. J. Lichtenberg, Principles of plasma discharges and materials processing, John Wiley \& Sons (2005).

[19] M. I. Boulos, P. Fauchasis, and E. Pfender, Thermal Plasmas:
Fundamentals and Applications, New York: Plenum Press (1994).

[20] K. S. Drellishak, C. F. Knopp, and A. B. Cambel, Phys. Fluids 6, 1280 (1963).

[21] D.-Y. Yang, Y. Kim, M. Y. Hur, H. J. Lee, Y.-J. Kim, T.-S. Lim, K.-B. Kim, and S. Yang, Metals 5, 2058-2069 (2015).

[22] M. Shiratani, H. Kawasaki, T. Fukuzawa, T. Yoshioka, Y. Ueda, S. Singh, and Y. Watanabe, J. Appl. Phys. 79, 104-109 (1996).

[23] R. L. Picard, A. H. Markosyan, D. H. Porter, S. L. Girshick, and M. J. Kushner, Plasma Chem. Plasma Process 36, 941-972 (2016).

[24] S.-W. Yoo, S.-J. You, J.-H. Kim, D.-J. Seong, B.-H. Seo, and N.M. Hwang, J. Phys. D: Appl. Phys. 50, 035201 (2017). 